\title{
Whole-mitochondrial genome sequencing in primary open-angle glaucoma using massively parallel sequencing identifies novel and known pathogenic variants
}

\author{
Periasamy Sundaresan, PhD ${ }^{1}$, David A. Simpson, PhD², Chitra Sambare, MBBS, FRCOphth ${ }^{2,3}$, \\ Seamus Duffy, BSc ${ }^{4}$, Judith Lechner, $\mathrm{PhD}^{2}$, Aditi Dastane, MBBS, MD ${ }^{5}$, \\ Edward W. Dervan, MD, FRCOphth ${ }^{6}$, Neeru Vallabh, MBBS7, Vidya Chelerkar, MBBS, MS ${ }^{3}$, \\ Madan Deshpande, MBBS, MS ${ }^{3}$, Colm O'Brien, MD, FRCOphth ${ }^{6}$, Amy Jayne McKnight, PhD ${ }^{4}$ \\ and Colin E. Willoughby, MD, FRCOphth ${ }^{7}$
}

\begin{abstract}
Purpose: The aim of this study was to determine whether mutations in mitochondrial DNA play a role in high-pressure primary openangle glaucoma (OMIM 137760) by analyzing new data from massively parallel sequencing of mitochondrial DNA.

Methods: Glaucoma patients with high-tension primary open-angle glaucoma and ethnically matched and age-matched control subjects without glaucoma were recruited. The entire human mitochondrial genome was amplified in two overlapping fragments by long-range polymerase chain reaction and used as a template for massively parallel sequencing on an Ion Torrent Personal Genome Machine. All variants were confirmed by conventional Sanger sequencing.

Results: Whole-mitochondrial genome sequencing was performed in 32 patients with primary open-angle glaucoma from India $(n=16)$ and Ireland $(n=16)$. In 16 of the 32 patients with primary openangle glaucoma ( $50 \%$ of cases), there were 22 mitochondrial DNA
\end{abstract}

mutations consisting of 7 novel mutations and 8 previously reported disease-associated sequence variants. Eight of 22 (36.4\%) of the mitochondrial DNA mutations were in complex I mitochondrial genes.

Conclusion: Massively parallel sequencing using the Ion Torrent Personal Genome Machine with confirmation by Sanger sequencing detected a pathogenic mitochondrial DNA mutation in 50\% of the primary open-angle glaucoma cohort. Our findings support the emerging concept that mitochondrial dysfunction results in the development of glaucoma and, more specifically, that complex I defects play a significant role in primary open-angle glaucoma pathogenesis.

Genet Med advance online publication 18 September 2014

Key Words: massively parallel sequencing; mitochondria; mtDNA; next-generation sequencing; primary open-angle glaucoma

\section{INTRODUCTION}

Glaucoma is a neurodegenerative disease of the eye and is one of the leading causes of visual impairment and blindness, affecting more than 60 million individuals worldwide. ${ }^{1}$ Primary open-angle glaucoma (POAG; OMIM 137760), the most common form of glaucoma, is defined as a spectrum of diseases causing vision loss as a result of progressive and irreversible retinal ganglion cell (RGC) damage, optic nerve degeneration, and corresponding visual field loss. ${ }^{2}$ POAG is usually associated with elevated intraocular pressure (IOP), and this subtype is termed high-tension glaucoma, defined as an IOP $>21 \mathrm{~mm} \mathrm{Hg}$ with a clinically open anterior chamber drainage angle. ${ }^{2}$ POAG accounts for $9-12 \%$ of all cases of blindness in the United States. ${ }^{3}$

Glaucoma is characterized by the slow, progressive degeneration of RGCs associated with structural changes at the optic nerve head in the form of pathological cupping and visual field loss. ${ }^{2,4}$ The preferential loss of RGCs in glaucoma is also a key pathological feature seen in Leber hereditary optic neuropathy and autosomal dominant optic atrophy. ${ }^{5,6}$ Mitochondrial abnormalities are associated with a number of optic neuropathies, ${ }^{5,6}$ and there is evidence that mitochondrial DNA (mtDNA) defects and dysfunction play a role in POAG. ${ }^{7-12}$ Mitochondrial genetic variants have also been linked to other neurodegenerative diseases, including Parkinson disease, Alzheimer disease, and Friedreich ataxia. ${ }^{13}$

The mitochondrial genome is organized as a circular, doublestranded DNA molecule in the mitochondrion and codes for only 37 genes across $\sim 16,600$ base pairs. ${ }^{14}$ mtDNA can replicate independently of nuclear DNA, and in humans there are usually 100-10,000 separate copies of mtDNA present in each cell. Mitochondrial mutations can be inherited through the maternal line or acquired throughout life (somatic mutations). Somatic mutations accumulate more frequently in the mitochondrial genome, which lacks the protective histones and efficient DNA repair system associated with the nuclear

The first two authors contributed equally to this work.

${ }^{1}$ Department of Genetics, Dr. G. Venkataswamy Eye Research Institute, Aravind Medical Research Foundation, Madurai, India; ${ }^{2}$ Centre for Experimental Medicine, Queen's

University Belfast, Belfast, UK; ${ }^{3}$ Poona Blind Men's Association's (PBMA's) H. V. Desai Eye Hospital, Pune, India; ${ }^{4}$ Centre for Public Health, Queen's University Belfast, Belfast, UK;

${ }^{5}$ Department of Genetics, Deenanth Mangeshkar Hospital, Pune, India; ${ }^{6}$ Institute of Ophthalmology, Mater Misericordiae University Hospital, Dublin, Ireland; ${ }^{7}$ Department of Eye and Vision Science, Institute of Ageing and Chronic Disease, University of Liverpool, Liverpool, UK. Correspondence: Colin E. Willoughby (c.willoughby@liverpool.ac.uk) 
genome $^{14,15}$ Mitochondria may contribute to their own accelerated DNA mutational rate during periods of localized environmental stress; mitochondria both generate and act as a target for excessive reactive oxygen species from altered oxidative phosphorylation. ${ }^{9}$ Several mtDNA variants can exist within a cell or tissue, a situation termed heteroplasmy. New mtDNA mutations can coexist at a low frequency with wildtype mtDNAs, making it challenging to identify all mutations in the mitochondrial genome. "Massively parallel" or "nextgeneration" sequencing approaches have ushered in a new era of opportunity in mtDNA mutation analysis, ${ }^{16,17}$ enhancing understanding of mtDNA-based disorders. ${ }^{18}$ Massively parallel sequencing can characterize the complete nucleotide sequence for the mitochondrial genome and provide adequate sequencing depth to identify heteroplasmic positions with enhanced sensitivity and estimate the mutant load at each nucleotide position. The aim of this study was to determine whether mutations in mtDNA play a role in POAG using massively parallel, wholemitochondrial genome sequencing on the Ion Torrent Personal Genome Machine (PGM; Life Technologies, Paisley, UK).

\section{MATERIALS AND METHODS}

\section{Patients}

Patients with high-tension POAG were recruited after careful phenotyping from the departments of ophthalmology in Dublin (Mater Misericordiae University Hospital, Dublin, Ireland) and Pune (PBMA's H. V. Desai Eye Hospital, Pune, India). Written informed consent was obtained from all subjects, and the study was performed according to the tenets of the Declaration of Helsinki and the guidelines of the Indian Council of Medical Research, New Delhi, India. All subjects underwent a standardized ophthalmic examination; the clinical parameters used for the case definition of high-tension POAG were as follows: (i) presenting IOP $>21 \mathrm{~mm} \mathrm{Hg}$ in at least one eye by Goldmann applanation tonometry; (ii) glaucomatous optic nerve head damage on stereoscopic optic disc examination (notching or thinning of the neuroretinal rim and/or increased cup/disc ratio in relation to the optic disc size); (iii) an open anterior chamber angle on gonioscopy; (iv) reproducible and characteristic glaucomatous visual field defect with the Humphrey 24-2 full-threshold strategy when available (Carl Zeiss Meditec, Oberkochen, Germany); and (v) absence of conditions leading to secondary glaucoma (e.g., pigmentary dispersion syndrome, pseudoexfoliation, history of steroid use, uveitis, or ocular trauma). Ethnically matched and age-matched control subjects without glaucoma (based on normal IOP) were also recruited from each study population.

\section{DNA Extraction and Polymerase Chain Reaction Amplification of Mitochondrial Genome}

Genomic DNA was extracted from peripheral blood leukocytes from all the subjects using a Qiagen DNA Purification Kit (Qiagen, Manchester, UK) according to the manufacturer's instructions. The entire human mitochondrial genome was amplified in two overlapping fragments: fragment 1 (5'-AACCAAACCCCAAAGACACC-3' ${ }^{\prime}$ and $5^{\prime}$-GCC AATAATGACGTGAAGTCC-3'; product size: 9,289 bp) and fragment 2 (5'-TCCCACTCCTAAACACATCC- ${ }^{\prime}$ and $5^{\prime}$-TTT ATGGGGTGATGTGAGCC-3'; product size: $7,626 \mathrm{bp}) .{ }^{16}$ Polymerase chain reaction (PCR) amplification was performed using a TaKaRa LA PCR Amplification Kit (Takara Bio, Shiga, Japan) in a $25-\mu$ l volume containing $0.125 \mu \mathrm{l}(5$ units/ $\mu \mathrm{l})$ of Taq DNA polymerase, $2.5 \mu \mathrm{l}$ of $10 \times$ Ex Taq buffer, $2 \mu \mathrm{l}$ deoxynucleotide triphosphates (dNTPs) $(2.5 \mathrm{mmol} / \mathrm{l} \mathrm{each})$, and $1 \mu \mathrm{mol} / \mathrm{l}$ primers. Thermal cycling was performed in a Verti thermal cycler (Life Technologies) with the following conditions: initial denaturation at $94{ }^{\circ} \mathrm{C}$ for $1 \mathrm{~min}$, followed by 35 cycles at $94{ }^{\circ} \mathrm{C}$ for $30 \mathrm{~s}, 64^{\circ} \mathrm{C}$ for $1 \mathrm{~min}$, and $72^{\circ} \mathrm{C}$ for $8 \mathrm{~min}$, and a final extension at $72^{\circ} \mathrm{C}$ for $5 \mathrm{~min}$. The amplified PCR products were purified using AMPure beads (Agencourt AMPure XP; Beckman Coulter, Danvers, MA) and quantified using a Nanodrop-1000 spectrophotometer (Nanodrop ND-1000; Thermo Scientific, Waltham, MA).

\section{Massively Parallel Sequencing on the lon Torrent PGM}

Massively parallel sequencing was performed on the Ion Torrent PGM according to the manufacturer's protocol (Life Technologies) and included library preparation, template preparation using emulsion PCR, and PGM sequencing. Specifically, Ion Torrent sequencing libraries were prepared using the Ion Xpress Fragment Plus Library Kit (Life Technologies) according to the manufacturer's protocol, with purification between steps performed using Agencourt AMPure XP beads (Beckman Coulter). First, equimolar amounts of mtDNA PCR fragments 1 and 2 were pooled for each sample and enzymatically sheared by incubation at $37^{\circ} \mathrm{C}$ for $15 \mathrm{~min}$ with Ion Shear enzyme (Life Technologies). After adaptor ligation (using Ion Xpress barcodes to uniquely identify each sample), the fragments were size-selected (150-250 bp) using an E-Gel SizeSelect $2 \%$ agarose gel (Life Technologies). The library was then amplified and quantified using a Qubit Fluorometer (Life Technologies). The library size of $\sim 200 \mathrm{bp}$ was verified using a 2100 Bioanalyzer (Agilent Technologies UK, Wokingham, UK). Clonal amplification of libraries on Ion Sphere particles was achieved by emulsion PCR performed on a One Touch system using the Ion OneTouch 100-bp Template Kit according to the manufacturer's instructions (Life Technologies). Template-positive Ion Sphere particles were isolated using the Ion OneTouch ES enrichment system. Massively parallel sequencing was performed on an Ion Torrent PGM instrument.

\section{PGM Data Analysis}

FASTQ output from the Ion Torrent was uploaded to Partek Flow for initial quality control and conversion to BAM files for analysis using Partek Genomics Suite version 6.6 (Partek, St. Louis, MO). The overall quality of reads was assessed using prealignment quality control with $10 \%$ of reads sampled per individual. Reads with less than 25 bases were removed and reads were trimmed until the quality score exceeded 20 . Trimmed data were independently aligned using Bowtie version 0.12.7 


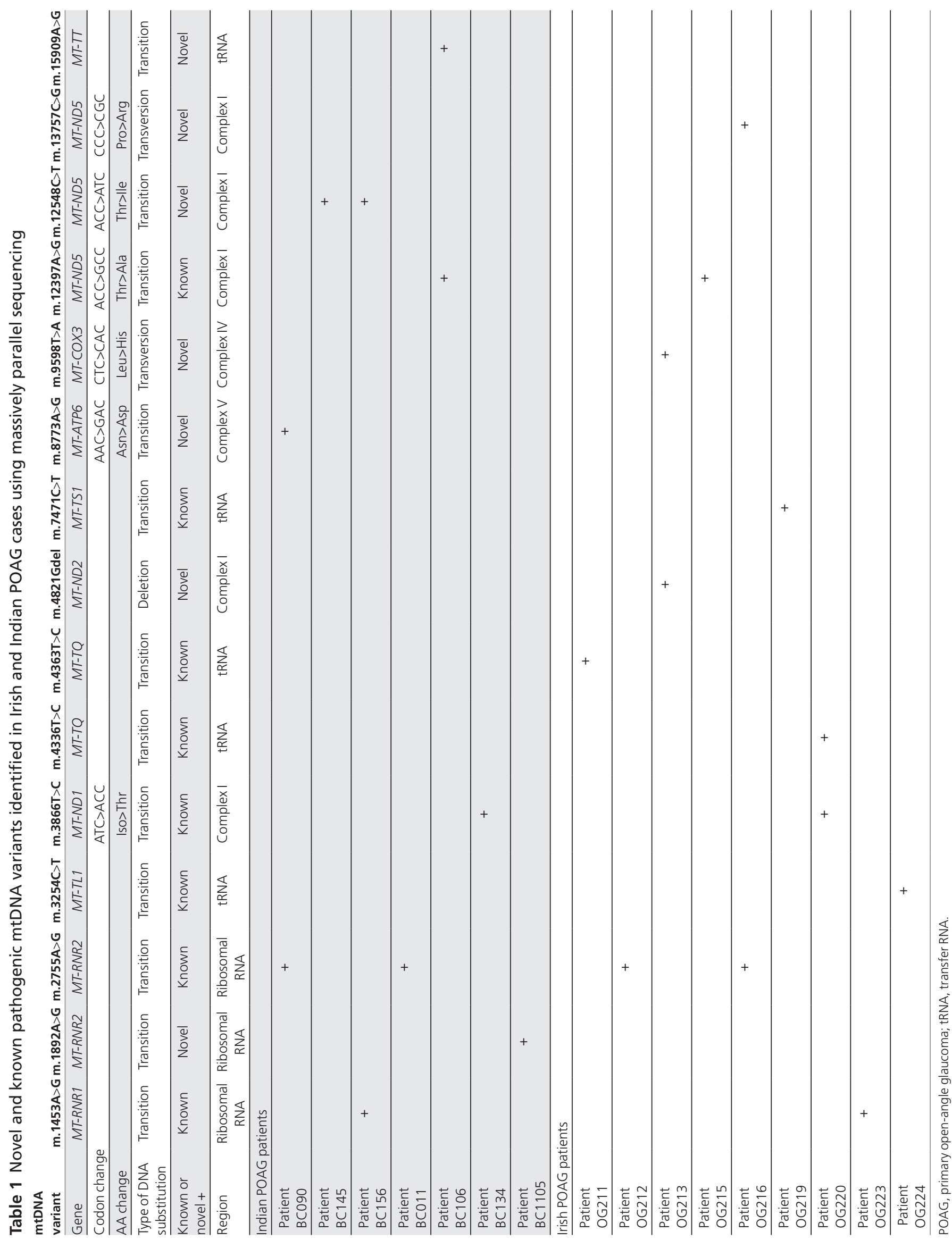

政 
(based on default settings optimized by Partek) to NCBI Reference mtDNA reference sequence (revised Cambridge Reference Sequence) NC_012920.1, GI:251831106 (hg19) with 0.2-bit reference built from FASTA sequence. mtDNA sequences for each sample were compared with the reference using single-nucleotide polymorphism (SNP) and allele percentage options.

\section{Sanger Sequencing of Mitochondrial Genome}

To corroborate the Ion Torrent PGM massively parallel sequencing, all patient mtDNA sequences and mtDNA from ethnically matched controls underwent conventional Sanger sequencing. Nine overlapping primer pairs were used for mtDNA amplification with 62 corresponding overlapping internal sequencing primers for mtDNA sequencing as previously described. . $^{19,20}$ Bidirectional cycle sequencing was performed using BigDye Terminator v3.1 cycle sequencing kit (Life Technologies), and amplified, targeted genetic regions electrophoresed on an ABI PRISM 3730 Genetic Analyzer (Life Technologies).

\section{mtDNA Variant Analysis}

Identified mtDNA sequence variants were compared with publicly accessible databases: MITOMAP (http://www.mitomap. org/MITOMAP), mtDB (http://www.mtdb.igp.uu.se/), dbSNP (Build 137) (http://www.ncbi.nlm.nih.gov/SNP/), and the 1000 Genomes Project (http://browser.1000genomes.org/index. html). Previously reported disease-associated sequence variants were identified using MITOMAP. Previously unreported or novel nucleotide changes were classified as pathogenic if they (i) were absent from ethnically matched controls (110 individuals without glaucoma), (ii) were not reported in the 24,187 human mitochondrial DNA sequences in MITOMAP, and (iii) were classified as possibly or probably damaging when considering the conservation and deleterious structural effects of amino acid substitutions on protein function using PolyPhen-2 (http://genetics.bwh.harvard.edu/pph2/). Identified sequence variants were described according to the guidelines published by the Human Genome Variation Society (http://www.hgvs. org). Transitions were represented by purine-purine $(A<->G)$ or pyrimidine-pyrimidine $(\mathrm{C}<->\mathrm{T})$ substitutions, and transversions were represented by purine-pyrimidine substitutions $(\mathrm{A}<->\mathrm{C}, \mathrm{A}<->\mathrm{T}, \mathrm{G}<->\mathrm{C}, \mathrm{G}<->\mathrm{T})$.

\section{RESULTS}

Whole-mitochondrial genome sequencing was performed in 32 patients with high-tension POAG from India $(n=16)$ and Ireland $(n=16)$ on the Ion Torrent PGM. Pre-alignment quality assurance and quality control on raw reads from the PGM (FASTQ files) demonstrated an average read length of 98 bases, with average quality score of 24 and $45 \%$ guanine cytosine content across the sequence reads. FASTA-trimmed read alignments demonstrated that more than $99.98 \%$ of mtDNA bases were covered from an average read length of 93 bases, with an average depth of $\times 267$. Unique sequence variants $(n$ $=536$ ) were identified in the Ion Torrent PGM sequencing data using the proportional allele model. Specifically, 334 Ion Torrent PGM sequence variants were confirmed by Sanger sequencing, whereas 57 were not confirmed by direct Sanger sequencing (likely false positives or below the heteroplasmy detection limit for Sanger sequencing), whereas the remainder were not able to be called by Sanger sequencing because of poor coverage, indels, or complex variations. Four hundred four unique SNPs were identified with log odds ratio $>5.0$ for the SNP-against-reference option in Partek. Eleven sequence variants were detected only by Sanger sequencing and were not identified by the algorithms used for analyzing the Ion Torrent PGM sequencing data (2.7\% false-negative rate). Sequenced variants detected in ethnically matched controls $(n=110)$ were excluded from further analysis.

Sixteen of the 32 POAG patients (50\% of cases) carried mtDNA sequence variants. We found a total of $22 \mathrm{mtDNA}$ sequence variants, consisting of seven novel mutations and eight previously reported disease-associated sequence variants (Table 1); six patients (18.75\%) harbored two pathogenic mtDNA mutations. Eight of $22(36.4 \%)$ mtDNA mutations were in complex I mitochondrial genes, and five of $22(22.7 \%)$ were in transfer RNAs. Among the POAG patients with mutations, five of $16(31.25 \%)$ had a mutation in the MT-ND5 gene in complex I. The eight previously reported disease-associated mtDNA sequence variants (Supplementary Table S1 online) were reported in MITOMAP. There was no evidence of a genotype-phenotype correlation between the number or location of mtDNA sequence variants and the severity of the POAG phenotype. None of the detected mtDNA sequence variants has previously been reported in POAG. Ten novel synonymous mtDNA variants were detected (Supplementary Table S2 online). In the Caucasian Irish POAG patients, nine of 16 (56.25\%) had at least one pathogenic mtDNA variant, and in the Indian POAG patients, seven of $16(43.75 \%)$ had at least one pathogenic mtDNA variant.

\section{DISCUSSION}

With a prevalence of $1-3 \%$ in Europe and up to $9 \%$ in Africa, glaucoma is a major cause of visual morbidity and the second most common cause of blindness in the world, resulting in bilateral blindness in 4.5 million people. ${ }^{1,21}$ Visual loss with POAG is the result of progressive and irreversible RGC damage. ${ }^{2}$ Given the high metabolic demands of RGCs, they are heavily dependent on mitochondrial function for their function and survival. ${ }^{9,22}$ Mitochondrial dysfunction and mtDNA defects have been implicated in RGC loss and the development of glaucoma. ${ }^{7,10,22,23}$

The expansion of massively parallel sequencing has revolutionized mitochondrial genetics. ${ }^{24-26}$ Sanger sequencing has long been considered the gold standard for molecular diagnosis of mtDNA-based disorders. The vast majority of deleterious mtDNA mutations are heteroplasmic, and it is challenging to identify all mutations in the mitochondrial genome and simultaneously quantify the mtDNA heteroplasmy levels. ${ }^{16}$ Previously, the two most popular complete mitochondrial 
genome sequencing methods were Sanger sequencing ${ }^{23}$ and the Affymetrix MitoChip ${ }^{27}$ but neither is sufficiently sensitive or specific to reliably detect mtDNA heteroplasmy. In comparison, massively parallel sequencing has been reported to detect mtDNA heteroplasmies of $\geq 5 \%$ with virtually no false-positive results. ${ }^{16,26,28}$ The application of massively parallel sequencing technology to the analysis of the mitochondrial genome has significantly improved the molecular diagnosis of mtDNA disorders. ${ }^{24}$ Large mtDNA deletions have been reported in mitochondrial diseases, and the ability of massively parallel sequencing to detect mtDNA deletions has not been firmly established..$^{29}$ However, using an long range PCR strategy similar to that in our study, the Ion Torrent PGM was able to detect two large-scale single deletions in the mtDNA of two patients. ${ }^{30}$

Two other studies have used massively parallel sequencing for whole-mitochondrial sequencing in POAG. Collins et al. ${ }^{31}$ used the Ion Torrent PGM to sequence the mitochondrial genome in 22 Afro-Caribbean POAG cases and 22 controls; they concluded that African-American POAG cases closely resembled controls with respect to frequency and types of mtDNA sequence variation. Using next-generation sequencing, Jeoung et al. ${ }^{32}$ identified three mtDNA SNPs $(\mathrm{m} .4883 \mathrm{C}>\mathrm{T}$ ND2, m.9540T>C COX3, and m.14766C $>\mathrm{T} C Y T B$ ) with significantly different frequencies in Korean patients with normal-tension glaucoma as compared with controls. In the present study, we evaluated 32 POAG patients using massively parallel sequencing on the Ion Torrent PGM to investigate the involvement of mitochondrial genomic variants in POAG pathogenesis and detected pathogenic mtDNA mutations in $50 \%$ of patients. The mutational load was consistent between the Irish Caucasian and Indian POAG patients, with complex I mitochondrial genes being the most commonly mutated, representing $36.4 \%$ of mutations. Our study is the first to assess mtDNA defects in Caucasian subjects with POAG. This mutational load is consistent with findings from Sanger sequencing of mtDNA in Middle Eastern Arabs with POAG that identified pathogenic mtDNA mutations in $54 \%$ of patients with POAG $(14 / 27) .{ }^{23}$ Mitochondrial mutations have also been reported in other forms of glaucoma at low frequencies: primary congenital glaucoma (10-22.85\%), ${ }^{33,34}$ primary angle closure glaucoma $(13.8 \%),{ }^{35}$ and pseudoexfoliation glaucoma (10.3\%). ${ }^{36}$ All previous mtDNA sequencing studies, including our study, were performed on mtDNA isolated from peripheral blood leukocytes, and it is known that mtDNA sequence variants may exist at different levels in different tissues (tissue heteroplasmy). Without studying mtDNA defects, including functional analyses, in the target tissue within the eye, these findings cannot be extrapolated to the site of disease pathology; however, they do provide evidence that mtDNA defects are frequently present in leukocytes of POAG patients, indicating that mitochondrial dysfunction is related to disease status.

Defects in complex I oxidative phosphorylation resulting in decreased respiration rates and adenosine triphosphate production have been reported in POAG lymphocytes, although this cohort was not screened for mtDNA defects. ${ }^{10}$ In our study, complex I was mutated in approximately one-third (31.25\%) of the POAG patients with mtDNA mutations and, specifically, mutations in the MT-ND5 gene. Banerjee et al. ${ }^{37}$ also observed a clustering of nonsynonymous mtDNA variants in MT-ND5 in a cohort of 101 patients with POAG from India evaluated by Sanger sequencing of the mitochondrial genome. Mitochondrial complex I is the largest component of the oxidative phosphorylation system and is a crucial component of the mitochondrial respiratory chain. ${ }^{38,39}$ The function of complex I is essential for the maintenance of tissue reactive oxygen species levels, generation of mitochondrial membrane potential, and adenosine triphosphate production. ${ }^{39}$ Complex I dysfunction leads to alterations in cell survival, apoptosis, and calcium homeostasis, and is the most frequent respiratory chain defect found in neuromuscular mitochondrial disorders. ${ }^{38}$ Genetic dysfunction of complex I is the most prevalent cause of Leber hereditary optic neuropathy, an inherited mitochondrial optic neuropathy ${ }^{40}$ Our study supports the emerging concept that mitochondrial dysfunction results in the development of glaucoma and, more specifically, that complex I and MT-ND5 defects play a significant role in POAG pathogenesis. ${ }^{7,22,37}$

Further studies using larger cohorts of glaucoma patients and phenotypes are required to determine the relationship between the mutational load in the mitochondrial genome and glaucoma pathogenesis. Massively parallel sequencing is a cost-effective approach with the high sensitivity, specificity, and accuracy necessary to detect a wide spectrum of mtDNA mutations while also quantifying levels of heteroplasmy. ${ }^{16,24,26,28}$ Without functional correlates, distinguishing mildly pathogenic mtDNA mutations from benign variants is challenging and can depend on interpretation. Future studies should correlate the mtDNA mutational spectrum with functional assays to more fully explore the role mitochondrial mutations and dysfunction play in the pathogenesis of glaucoma.

\section{SUPPLEMENTARY MATERIAL}

Supplementary material is linked to the online version of the paper at http://www.nature.com/gim.

\section{ACKNOWLEDGMENTS}

This study was partially funded by the British Council for the Prevention of Blindness. C.S. was the recipient of the Sir John Wilson Prevention of Blindness Fellowship. P.S. received funding from the Department of Biotechnology, Government of India (CREST award 2010-2011). We thank the subjects who took part in this research.

\section{DISCLOSURE}

The authors declare no conflict of interest.

\section{REFERENCES}

1. Quigley HA, Broman AT. The number of people with glaucoma worldwide in 2010 and 2020. Br J Ophthalmol 2006;90:262-267.

2. Weinreb RN, Khaw PT. Primary open-angle glaucoma. Lancet 2004;363: 1711-1720. 
3. Quigley HA, Vitale S. Models of open-angle glaucoma prevalence and incidence in the United States. Invest Ophthalmol Vis Sci 1997;38:83-91.

4. Osborne NN, del Olmo-Aguado S. Maintenance of retinal ganglion cell mitochondrial functions as a neuroprotective strategy in glaucoma. Curr Opin Pharmacol 2013;13:16-22.

5. Yu-Wai-Man P, Griffiths PG, Hudson G, Chinnery PF. Inherited mitochondrial optic neuropathies. J Med Genet 2009;46:145-158.

6. Carelli V, Ross-Cisneros FN, Sadun AA. Mitochondrial dysfunction as a cause of optic neuropathies. Prog Retin Eye Res 2004;23:53-89.

7. Lascaratos G, Garway-Heath DF, Willoughby CE, Chau KY, Schapira AH. Mitochondrial dysfunction in glaucoma: understanding genetic influences. Mitochondrion 2012:12:202-212.

8. He Y, Ge J, Tombran-Tink J. Mitochondrial defects and dysfunction in calcium regulation in glaucomatous trabecular meshwork cells. Invest Ophthalmol Vis Sci 2008;49:4912-4922.

9. Chrysostomou V, Rezania F, Trounce IA, Crowston JG. Oxidative stress and mitochondrial dysfunction in glaucoma. Curr Opin Pharmacol 2013;13:12-15.

10. Lee S, Sheck L, Crowston JG, et al. Impaired complex-I-linked respiration and ATP synthesis in primary open-angle glaucoma patient lymphoblasts. Invest Ophthalmol Vis Sci 2012;53:2431-2437.

11. Noh YH, Kim KY, Shim MS, et al. Inhibition of oxidative stress by coenzyme Q10 increases mitochondrial mass and improves bioenergetic function in optic nerve head astrocytes. Cell Death Dis 2013;4:e820.

12. McElnea EM, Quill B, Docherty NG, et al. Oxidative stress, mitochondrial dysfunction and calcium overload in human lamina cribrosa cells from glaucoma donors. Mol Vis 2011;17:1182-1191.

13. Chaturvedi RK, Flint Beal M. Mitochondrial diseases of the brain. Free Radic Biol Med 2013;63:1-29.

14. Druzhyna NM, Wilson GL, LeDoux SP. Mitochondrial DNA repair in aging and disease. Mech Ageing Dev 2008;129:383-390.

15. Larsen NB, Rasmussen M, Rasmussen $\sqcup$. Nuclear and mitochondrial DNA repair: similar pathways? Mitochondrion 2005;5:89-108.

16. Tang $S$, Huang T. Characterization of mitochondrial DNA heteroplasmy using a parallel sequencing system. BioTechniques 2010;48:287-296.

17. Li M, Schönberg A, Schaefer M, Schroeder R, Nasidze I, Stoneking M. Detecting heteroplasmy from high-throughput sequencing of complete human mitochondrial DNA genomes. Am J Hum Genet 2010;87:237-249.

18. Zaragoza MV, Fass J, Diegoli M, Lin D, Arbustini E. Mitochondrial DNA variant discovery and evaluation in human cardiomyopathies through next-generation sequencing. PLOS ONE 2010;5:e12295.

19. Ramos A, Santos C, Alvarez L, Nogués R, Aluja MP. Human mitochondrial DNA complete amplification and sequencing: a new validated primer set that prevents nuclear DNA sequences of mitochondrial origin co-amplification. Electrophoresis 2009;30:1587-1593.

20. Ramos A, Santos C, Barbena E, et al. Validated primer set that prevents nuclear DNA sequences of mitochondrial origin co-amplification: a revision based on the New Human Genome Reference Sequence (GRCh37). Electrophoresis 2011;32:782-783.

21. Leske MC. Open-angle glaucoma - an epidemiologic overview. Ophthalmic Epidemio/ 2007;14:166-172.
22. Lee S, Van Bergen NJ, Kong GY, et al. Mitochondrial dysfunction in glaucoma and emerging bioenergetic therapies. Exp Eye Res 2011;93:204-212.

23. Abu-Amero KK, Morales J, Bosley TM. Mitochondrial abnormalities in patients with primary open-angle glaucoma. Invest Ophthalmol Vis Sci 2006;47:25332541.

24. Calvo SE, Compton AG, Hershman SG, et al. Molecular diagnosis of infantile mitochondrial disease with targeted next-generation sequencing. Sci Transl Med 2012;4:118ra110.

25. Vasta V, Ng SB, Turner EH, Shendure J, Hahn SH. Next generation sequence analysis for mitochondrial disorders. Genome Med 2009;1:100.

26. Cui H, Li F, Chen D, et al. Comprehensive next-generation sequence analyses of the entire mitochondrial genome reveal new insights into the molecular diagnosis of mitochondrial DNA disorders. Genet Med 2013;15:388-394.

27. van Eijsden RG, Gerards M, Eijssen LM, et al. Chip-based mtDNA mutation screening enables fast and reliable genetic diagnosis of OXPHOS patients. Genet Med 2006;8:620-627.

28. Tang S, Wang J, Zhang VW, et al. Transition to next generation analysis of the whole mitochondrial genome: a summary of molecular defects. Hum Mutat 2013;34:882-893.

29. Kingsley CB. Identification of causal sequence variants of disease in the next generation sequencing era. Methods Mol Biol 2011;700:37-46.

30. Seneca S, Vancampenhout K, Van Coster R, et al. Analysis of the whole mitochondrial genome: translation of the Ion Torrent Personal Genome Machine system to the diagnostic bench? Eur J Hum Genet 2014; e-pub ahead of print 26 March 2014.

31. Collins DW, Gudiseva HV, Trachtman BT, et al. Mitochondrial sequence variation in African-American primary open-angle glaucoma patients. PLOS ONE 2013;8:e76627.

32. Jeoung JW, Seong MW, Park SS, Kim DM, Kim SH, Park KH. Mitochondrial DNA variant discovery in normal-tension glaucoma patients by next-generation sequencing. Invest Ophthalmol Vis Sci 2014;55:986-992.

33. Tanwar M, Dada T, Sihota R, Dada R. Mitochondrial DNA analysis in primary congenital glaucoma. Mol Vis 2010;16:518-533.

34. Kumar M, Tanwar M, Faiq MA, et al. Mitochondrial DNA nucleotide changes in primary congenital glaucoma patients. Mol Vis 2013;19:220-230.

35. Abu-Amero KK, Morales J, Osman MN, Bosley TM. Nuclear and mitochondrial analysis of patients with primary angle-closure glaucoma. Invest Ophthalmol Vis Sci 2007; 48:5591-5596.

36. Abu-Amero KK, Bosley TM, Morales J. Analysis of nuclear and mitochondrial genes in patients with pseudoexfoliation glaucoma. Mol Vis 2008;14:29-36.

37. Banerjee $D$, Banerjee $A$, Mookherjee $S$, et al. Mitochondrial genome analysis of primary open angle glaucoma patients. PLOS ONE 2013;8:e70760.

38. Papa S, De Rasmo D. Complex I deficiencies in neurological disorders. Trends Mol Med 2013;19:61-69.

39. Roestenberg P, Manjeri GR, Valsecchi F, Smeitink JA, Willems PH, Koopman WJ. Pharmacological targeting of mitochondrial complex I deficiency: the cellular level and beyond. Mitochondrion 2012;12:57-65.

40. Yu-Wai-Man P, Griffiths PG, Chinnery PF. Mitochondrial optic neuropathies disease mechanisms and therapeutic strategies. Prog Retin Eye Res 2011;30:81114. 